N. TEZUKA, K. Shimojo, K. HiRAno,* S. KOMAGAWA, K. YOShidA, C. WANG,

K. MiYAmOTO, T. SAITO, R. TAKITA, M. UCHIYAMA* (THE UNIVERSITY OF TOKYO AND

RIKEN CENTER FOR SUSTAINABLE RESOURCE SCIENCE, SAITAMA, JAPAN)

Direct Hydroxylation and Amination of Arenes via Deprotonative Cupration

J. Am. Chem. Soc. 2016, 138, 9166-9171.

\title{
Aromatic Hydroxylation and Amination through Deprotonative Cupration
}

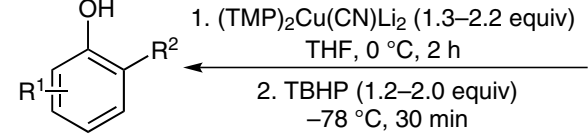

L

$\mathrm{R}^{1}=\mathrm{H}, \mathrm{Cl}, \mathrm{Br}, \mathrm{I}, \mathrm{CF}_{3}, \mathrm{All}, \mathrm{Ph}$, Alk, OMe $\mathrm{R}^{2}=\mathrm{CON}(\mathrm{Alk})_{2}, \mathrm{CO}_{2} \mathrm{Alk}, \mathrm{CO}_{2} \mathrm{H}, \mathrm{CN}, \mathrm{OMe}, \mathrm{OMOM}, \mathrm{POCy}$

\section{Category}

Metal-Mediated

Synthesis

\section{Key words}

hydroxylation

amination

cupration

Selected examples:

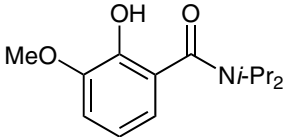

$79 \%$ yield

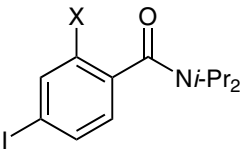

$\mathrm{X}=\mathrm{NH}_{2}: 84 \%$ yield $\mathrm{X}=\mathrm{OH}: 92 \%$ yield<smiles>Nc1nccc2ccccc12</smiles>

$87 \%$ yield<smiles>[X]c1cc(C(C)(C)C)ccc1C#N</smiles>

$\mathrm{X}=\mathrm{NH}_{2}: 84 \%$ yield $\mathrm{X}=\mathrm{OH}: 87 \%$ yield

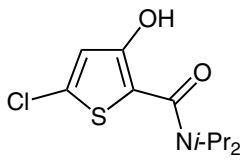

$81 \%$ yield<smiles>Nc1cc(-c2ccccc2)oc1C(=O)NC(=O)c1ccccc1</smiles>

$46 \%$ yield<smiles>[X]c1ccccc1P(=O)=O</smiles>

$\mathrm{X}=\mathrm{NH}_{2}: 94 \%$ yield $\mathrm{X}=\mathrm{OH}: 86 \%$ yield<smiles>[X]c1ccc2ccccc2c1C(=O)NCCCC</smiles>

$\mathrm{X}=\mathrm{NH}_{2}: 76 \%$ yield $\mathrm{X}=\mathrm{OH}: 89 \%$ yield



$63 \%$ yield<smiles></smiles>

16 examples up to $94 \%$ yield
Significance: The authors report an efficient aromatic hydroxylation and amination reaction through directed ortho cupration. The method is applicable to a wide range of functionalized aromatic and heteroaromatic compounds, and shows high chemo- and regioselectivity.
Comment: The reported reaction is the first example of an efficient, one-pot synthesis of functionalized phenols and anilines from the same substrates.

SYNFACTS Contributors: Paul Knochel, Marthe Ketels 